\title{
High Angular Accuracy EBSD based on a 3D Hough Transform
}

\author{
Claire Maurice ${ }^{1}$, Austin Day ${ }^{2}$ and Roland Fortunier ${ }^{3}$ \\ 1. Ecole des Mines, Laboratory Georges Friedel, CNRS UMR 5307, Saint-Etienne, France \\ 2. Aunt Daisy Scientific Ltd, Claremont House, Lydney, United Kingdom \\ 3. Université de Lyon, ENISE, CNRS UMR 5513 LTDS, Saint-Etienne, France
}

A novel procedure for high angular accuracy Electron Backscatter Diffraction (HAA-EBSD) is presented. It allows accurate localisation of Kikuchi bands by means of a local 3-dimensional Hough transform (extended from that proposed in [1]) and will determine, simultaneously, the pattern centre, crystal orientation and lattice strain. After validation on dynamically simulated patterns, it has been applied to experimental electron backscatter patterns (EBSP). Several practical difficulties have been investigated, for example lens distortions [2], and solutions implemented.

There are many applications for this new technique, for example improved phase discrimination / identification, better EBSP orientation measurement... The most topical application is, in combination with the cross-correlation technique [3-5], to analyse a reference EBSP to determine stress state and orientation - this could allow "absolute" stress analysis at the local scale.

EBSD is now a standard tool for characterising microstructures and microtextures. Since the pioneering work of Troost et al. in the early 1990s and further developments by Wilkinson et al., and others, EBSD is also increasingly used for elastic strain measurements at the local scale or plastic deformation analysis via GND characterisation [e.g. 3-5]. The "cross-correlation" or "pattern shift" method for EBSD elastic strain analysis, finds the displacement field between a reference and a selected EBSP, which is then interpreted as lattice strain and rigid body rotation. However, in many cases, e.g. for polycrystalline samples, a reference pattern with known strain state may not be available, and cross-correlation then produces relative rather than absolute strain data. This new procedure may overcome this limitation.

The 3D-Hough transform procedure is schematically explained in Figure 1. The accumulative Hough space is constructed as a 3D space where the (x-axis, y-axis) base plane is parameterized to depict the unit normal of a given reflector, while the Bragg angle is represented on the z-axis. For a given reflector, physically plausible Kossel cone projections are searched for in a small sub-volume around its approximate position, derived from standard indexing. The algorithm works as follows: assuming a source point position $\left(X_{s}, Y_{s}, Z_{s}\right)$, each pixel $(x, y)$ of the EBSP is related to a normalized wave vector through the gnomonic projection

$\vec{u}=\left[\left(x-X_{s}\right)^{2}+\left(y-Y_{s}\right)^{2}+Z_{s}{ }^{2}\right]\left(x-X_{s} \quad y-Y_{s}-Z_{s}\right)^{T}$. Then, each cell $\left(n_{1}, n_{2}\right)$ of the Hough plane is used to calculate a unit normal vector $\vec{n}$, from which the dot product with $\vec{u}, \vec{u} . \vec{n}=\sin \theta_{B}$ is computed. The intensity of pixel $(x, y)$ is then added to the Hough voxel $\left(n_{1}, n_{2}, \sin \theta_{B}\right)$. The peak shape shown in Fig 1c reveals an intensity maximum located at the representative point of the examined hyperbola, thus allowing the determination of the reflector normal with an increased accuracy compared to standard 2D Hough transform [1]. From accurately determined normal vectors, standard algorithms are employed to derive the crystal orientation and lattice strain [1].

It is obvious from the algorithm described above that the source point position (or Pattern Centre PC $\left(\mathrm{X}_{\mathrm{s}}, \mathrm{Y}_{\mathrm{s}}\right)$ and Detector Distance $\mathrm{Z}_{\mathrm{s}}=\mathrm{DD}$ ) used as an input, directly influences the peak intensity distribution in the Hough box. Using dynamically simulated patterns (in-house software developed 
following Winkelmann et al. [6]) it can be shown that the peak morphology is affected when the input source point is shifted with respect to its true value, as shown in Figure 2. In fact, a wrong PC (a and c) gives a horizontally sheared peak, while a wrong DD ( $d$ and f) produces a vertical asymmetry of the peak. This behaviour is quantified by means of two parameters describing the peak morphology, the first being primarily sensitive to PC, while the second varies to first order with DD. As illustrated in figure $2 \mathrm{~g}$ ), scanning the Pattern Centre space will give for each reflector a smooth linear variation of the morphological parameter, where the white line perpendicular to the reflector gives the likely positions of the PC. Summing over several reflectors yields figure $2 \mathrm{~h}$ ) or $2 \mathrm{i}$ ) in 3D which shows a single well defined minimum located at the true PC position.

This new method will be explained and its potential applications illustrated with experimental data.

References:

[1] C. Maurice and R. Fortunier, Journal of Microscopy 230 (2008) 520-529.

[2] K. Mingard et al., Ultramicroscopy 111 (2011) 320-329.

[3] K.Z. Troost, P. van der Sluis and D.J. Gravesteijn, Appl. Phys. Lett. 62 (1993) 1110-1112.

[4] A.J. Wilkinson, G. Meaden and D.J. Dingley, Ultramicroscopy 106 (2006) 307-313.

[5] S. Villert et al., Journal of Microscopy 233 (2009) 290-301.

[6] A. Winkelmann et al., Ultramicroscopy 107 (2007) 414-421.

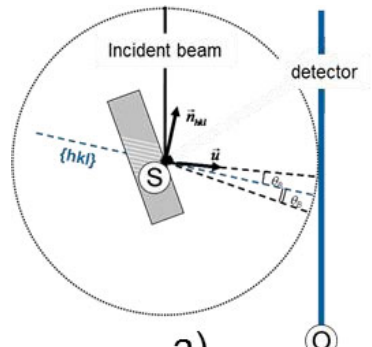

a)

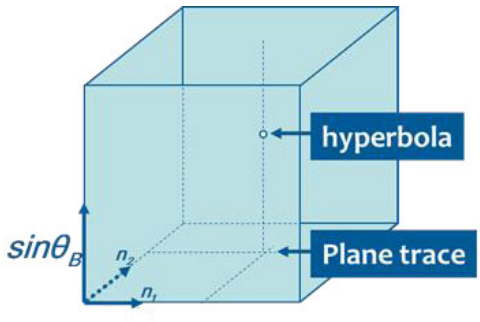

b)

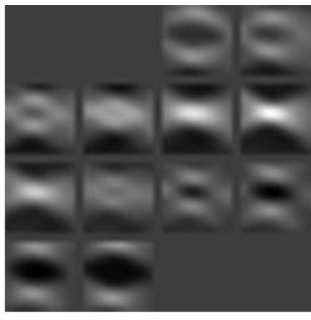

c)

Figure 1. Schematic of the local 3D Hough transform a) the EBSD geometry, b) parameterization of the $3 \mathrm{D}$ Hough space, c) typical Hough peak shown in $\sin \theta_{B}$ constant sections.
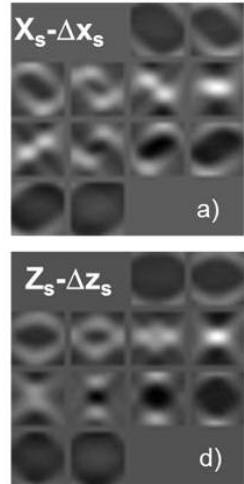
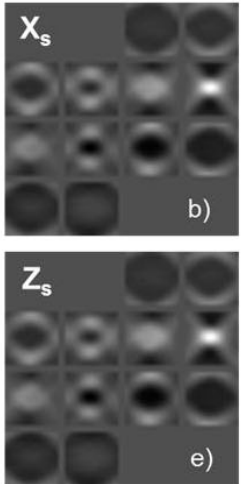
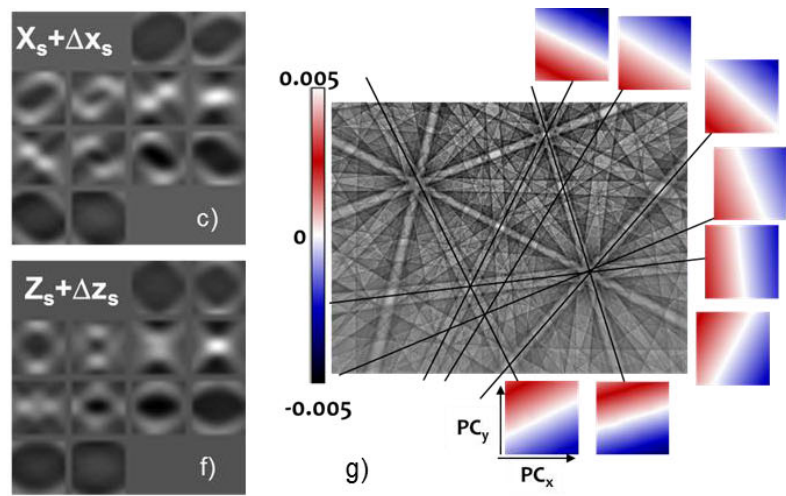
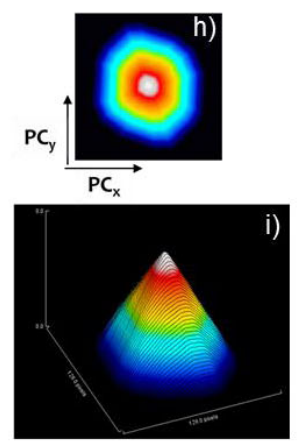

Figure 2. Influence of the input Pattern Centre on the 3D Hough peak morphology. b) and e) peak calculated from true PC, a) and c) effect of a wrong PC, d) and f) effect of a wrong DD, g) maps of the first morphological parameter in PC space for a set of reflectors, h) and i) summation of the first morphological parameter over set of reflectors in g), showing the existence of a single well defined minimum located at the true PC position. 